Article

\title{
Correction of the Koenig formula for the kinetic energy of a rotating solid
}

\author{
Yuriy Alyushin \\ National Research Technological University MISiS, Moscow, 119991, Russia \\ alyushin7@gmail.com; Tel.: +79162218591
}

\begin{abstract}
An exact solution is obtained for the kinetic energy in the general case of the spatial motion of solids with arbitrary rotation, which differs from the Koenig formula by three additional terms that take into account the change in the centrifugal moments of inertia when the body rotates. The description of motion in the Lagrange form and the superposition principle are used, which provides a geometric summation of the velocities and accelerations of the joint motions in the Lagrange form for any particle at any time. The integrand function in the equation for kinetic energy is represented as the sum of the identical velocity components of the joint plane-parallel motions. In the general case of motion with 6 degrees of freedom, the energy of rotational motion is determined by three axial moments of inertia, as in the Koenig formula, and three additional centrifugal moments, which take into account the rotation of the body. They can be calculated through 6 integral characteristics of the density distribution, determined for the initial position of the body.
\end{abstract}

Keywords: kinetic energy, Lagrange variables, the principle of superposition of motions, axial and centrifugal moments of inertia.

\section{Introduction}

Kinetic energy is a mandatory and usually the main component of the motion of absolutely rigid bodies. This requires special responsibility when choosing methods for determining it, for example, in the dynamic analysis of mobile robots and other mechanisms, the complexity of which increases with the development of technological progress.

The most popular formula Koenig [1-2] for spatial motion

$$
E_{k}=0,5 m v_{C}^{2}+0,5\left(\theta_{x, t}^{2} J_{C}^{x}+\theta_{y, t}^{2} J_{C}^{y}+\theta_{z, t}^{2} J_{C}^{z}\right),
$$

where $\theta_{i, t}, J_{C}^{i}$ is the angular velocities and moments of inertia of the body relative to the central axes, was published in 1751 [2]. The basis is taken the assumption of the equality of the kinetic energy of a material system the amount of energy a portable translational motion along with the center of mass and the energy of rotational motion relative to axes moving with the center of mass. Given that the movements of each particle when joint plane-parallel rotations form simultaneously rotations in two planes, there is reason to assume that the sum of the last three terms exceeds the actual kinetic energy of the joint motion.

To clarify the kinetic energy determined by the rotational motion of a body in formula (1), we use the general form of the equations of motion in the space of Lagrange variables [3-4]. By the current coordinates $x_{i} \in(x, y, z)$, we will use the initial coordinates of the particles $\alpha_{p}=\left.x_{p}\right|_{t=0}$ for the Lagrange variables $\alpha_{p} \in(\alpha, \beta, \gamma)$. 
Consider the rotation of a body in the $x-y$ plane with respect to the fixed pole $P\left(\alpha_{P}, \beta_{P}\right)$. The initial position of an arbitrary point $M(\alpha, \beta)$ is determined by the angle $\left(\theta_{z}\right)_{0}$ of inclination of the straight-line $P M$ with respect to the $\mathrm{x}$-axis and the distance between the points $|P M|=L=$ const

$$
\alpha=\alpha_{P}+L \cos \left(\theta_{z}\right)_{0}, \quad \beta=\beta_{P}+L \sin \left(\theta_{z}\right)_{0} .
$$

After turning the straight-line $P M$ by an angle $\Delta \theta_{x}=\theta_{x}-\left(\theta_{x}\right)_{0}$, the coordinates of the point can be determined by the same relations,

$$
x=\alpha_{P}+L \cos \theta_{z}=\alpha_{P}+L \cos \left[\left(\theta_{z}\right)_{0}+\Delta \theta_{z}\right], \quad y=\beta_{P}+L \sin \left[\left(\theta_{z}\right)_{0}+\Delta \theta_{z}\right] .
$$

Excluding from these equations the length $\mathrm{L}$ and the initial value of the angle $\left(\theta_{x}\right)_{0}$ using equations (2), we obtain a system of equations of motion,

$$
x=\alpha_{P}+\left(\alpha-\alpha_{P}\right) \cos \Delta \theta_{z}-\left(\beta-\beta_{P}\right) \sin \Delta \theta_{z}, \quad y=\beta_{P}+\left(\alpha-\alpha_{P}\right) \sin \Delta \theta_{z}+\left(\beta-\beta_{P}\right) \cos \Delta \theta_{z}, \quad z=\gamma
$$

and the corresponding speeds

$$
x_{t}=-\theta_{z, t}\left(y-\beta_{P}\right), \quad y_{t}=\theta_{z, t}\left(x-\alpha_{P}\right), \quad z_{t}=0 .
$$

The lower index $t$ when denoting the coordinates $x_{i, t}$ and angles of rotation $\theta_{i, t}$ corresponds to the differentiation of the corresponding functions $d x_{i} / d t \equiv x_{i, t}, d \theta_{i} / d t \equiv \theta_{i, t}$.

For plane-parallel movements in other planes, the equations can be obtained by using a circular substitution: when rotating relative to the $x$-axis:

$$
\begin{gathered}
x=\alpha, \quad x_{t}=0, \\
y=\beta_{P}+\left(\beta-\beta_{P}\right) \cos \Delta \theta_{x}-\left(\gamma-\gamma_{P}\right) \sin \Delta \theta_{x}, y_{t}=-\theta_{x, t}\left(z-\gamma_{P}\right), \\
z=\gamma_{P}+\left(\beta-\beta_{P}\right) \sin \Delta \theta_{x}+\left(\gamma-\gamma_{P}\right) \cos \Delta \theta_{x}, z_{t}=\theta_{x, t}\left(y-\beta_{P}\right),
\end{gathered}
$$

when rotating relative to the $y$-axis:

$$
\begin{array}{rc}
x=\alpha_{P}+\left(\alpha-\alpha_{P}\right) \cos \Delta \theta_{y}+\left(\gamma-\gamma_{P}\right) \sin \Delta \theta_{y}, & x_{t}=\theta_{y, t}\left(z-\gamma_{P}\right), \\
y=\beta, & y_{t}=0, \\
z=\gamma_{P}+\left(\gamma-\gamma_{P}\right) \cos \Delta \theta_{y}-\left(\alpha-\alpha_{P}\right) \sin \Delta \theta_{y}, & z_{t}=-\theta_{y, t}\left(x-\alpha_{P}\right) .
\end{array}
$$

The lower index when indicating the angle of rotation of the body $\theta_{i}$ indicates the direction of the axis of rotation passing through the pole $P$ parallel to the axes of the observer's coordinate system, relative to which the body rotates. Equations (3) - (6) will be used as the initial ones in all further transformations.

For spatial movements, systems of type (6) can be obtained using the superposition principle [5], which allows us to consider complex spatial processes as the simultaneous realization of several movements. Since the Euler and Lagrange variables coincide in the initial state $x_{i}\left(\alpha_{p}, t=0\right)=\alpha_{i}$, the joint of movements is reduced to replacing 
the Lagrange variables of the external motion $x_{i}^{e x}=x_{i}^{e x}\left(\alpha_{p}, t\right)$ with expressions for the corresponding Euler variables of the internal motion $x_{i}^{i n}=x_{i}^{i n}\left(\alpha_{p}, t\right)$. The equations of joint motion $x_{i}=x_{i}\left(\alpha_{p}, t\right)$ in their sequential or simultaneous flow coincide with the equations of external motion after replacing the Lagrange variables with the corresponding equations for the Euler variables of internal motion,

$$
x_{i}\left(\alpha_{p}, t\right)=x_{i}^{e x}\left(x_{i}^{i n}\left(\alpha_{p}, t\right), t\right) .
$$

External and internal movements are similar to figurative and relative ones in classical mechanics, but differ by the mandatory use of a single coordinate system. The principle allows its repeated application without violating the rule of geometric addition of the velocities and accelerations of the joint motion at each point and at each moment of time [5-6].

For example, to obtain the equations of motion with rotation relative to a moving pole, one should superimpose on the rotations (3) - (6) the translational displacement of the pole $P$,

$$
x=\alpha+x_{P}(t)-\alpha_{P}, \quad y=\beta+y_{P}(t)-\beta_{P}, \quad z=\gamma+z_{P}(t)-\gamma_{P} .
$$

As a result, the first term (Lagrangian coordinate) in each of the equations (3) - (6) is replaced by the current (Eulerian) coordinate

$$
\begin{array}{cc}
x=x_{P}+\left(\alpha-\alpha_{P}\right) \cos \Delta \theta_{z}-\left(\beta-\beta_{P}\right) \sin \Delta \theta_{z}, \quad y=y_{P}+\left(\alpha-\alpha_{P}\right) \sin \Delta \theta_{z}+\left(\beta-\beta_{P}\right) \cos \Delta \theta_{z}, \quad z=\gamma+z_{P}-\gamma_{P}, \\
x=\alpha+x_{P}-\alpha_{P}, \quad y=y_{P}+\left(\beta-\beta_{P}\right) \cos \Delta \theta_{x}-\left(\gamma-\gamma_{P}\right) \sin \Delta \theta_{x}, \quad z=z_{P}+\left(\beta-\beta_{P}\right) \sin \Delta \theta_{x}+\left(\gamma-\gamma_{P}\right) \cos \Delta \theta_{x}, \\
x=x_{P}+\left(\alpha-\alpha_{P}\right) \cos \Delta \theta_{y}+\left(\gamma-\gamma_{P}\right) \sin \Delta \theta_{y}, \quad y=\beta+y_{P}-\beta_{P}, \quad z=z_{P}+\left(\gamma-\gamma_{P}\right) \cos \Delta \theta_{y}-\left(\alpha-\alpha_{P}\right) \sin \Delta \theta_{y} .
\end{array}
$$

Each system of 3 equations corresponds to a motion with 4 degrees of freedom: 3 translational movements along the coordinate axes of the pole $\mathrm{P}$ and the rotation of the body $\Delta \theta_{i}$ relative to the corresponding axis.

Equations (3) - (9) are sufficient to determine any kinematic characteristics of the motion of absolutely rigid bodies, including the kinetic energy, which, by definition, for a body with volume $V$, density $\rho$ and mass $m$, is determined by the integral [1-2]

$$
E_{k}=0,5 \rho \int_{V} v^{2} \delta V=0,5 \int_{m} v^{2} \delta m=0,5 \int_{m}\left(x_{t}^{2}+y_{t}^{2}+z_{t}^{2}\right) \delta m .
$$

Taking into account the different nature of the arguments when describing motion in the Lagrange form, in equation (10) and below, the operator $\delta$ is used to denote infinitesimal increments of arguments and functions in the space of Lagrange variables, including when integrating with respect to mass $\delta m$. The $d$ operator characterizes infinitesimal changes in functions over time, as used above for the velocity components $d x_{i} / d t \equiv x_{i, t}$.

\section{Correction of the Koenig formula}

The exact integration of equation (10) can be performed for plane-parallel motion with rotation relative to one axis, for example, $z$, when over the entire volume of the body $z_{t}=0$. Taking into account the equations of motion (9), we transform the right part to the form

$$
E_{k}=0,5 m\left[\left(x_{t}\right)_{P}^{2}+\left(y_{t}\right)_{P}^{2}\right]-\theta_{z, t} \int_{m}\left[\left(x_{t}\right)_{P}\left(y-y_{P}\right)-\left(y_{t}\right)_{P}\left(x-x_{P}\right)\right] \delta m+0,5 \theta_{z, t}^{2} \int_{m}\left[\left(x-x_{P}\right)^{2}+\left(y-y_{P}\right)^{2}\right] \delta m .
$$


For integration in the second term, we use the concept of the center of mass $C\left(x_{c}, y_{c}\right)$, whose coordinates [1-2] define the equations

$$
x_{C} m=\int_{m} x \delta m, \quad y_{C} m=\int_{m} y \delta m
$$

Since $x_{C}$ and $y_{c}$ remain the same for all particles, they can be extracted from the integral and written instead of (11)

$$
E_{k}=0,5 m\left[\left(x_{t}\right)_{P}^{2}+\left(y_{t}\right)_{P}^{2}\right]-\theta_{z, t} m\left[\left(x_{t}\right)_{P}\left(y_{C}-y_{P}\right)-\left(y_{t}\right)_{P}\left(x_{C}-x_{P}\right)\right]+0,5 \theta_{z, t}^{2} \int_{m}\left[\left(x-x_{P}\right)^{2}+\left(y-y_{P}\right)^{2}\right] \delta m \text {. }
$$

The integral remaining in the right part is a measure of the inertia of a body in rotational motion, by analogy with the mass $m$ in translational motion,

$$
J_{P}^{z}=\int_{m}\left[\left(x-x_{P}\right)^{2}+\left(y-y_{P}\right)^{2}\right] \delta m
$$

As a result, instead of (13) we get

$$
E_{k}=0,5 m\left(x_{t}^{2}+y_{t}^{2}\right)_{P}+0,5 \theta_{z, t}^{2} J_{P}-\theta_{z, t} m\left[\left(x_{t}\right)_{P}\left(y_{C}-y_{P}\right)-\left(y_{t}\right)_{P}\left(x_{C}-x_{P}\right)\right] .
$$

The position of the center of mass depends on the configuration of the body and is determined by equations (12). The position of the pole can be selected arbitrarily. If the center of mass is chosen as the pole, the last term turns to 0 and the formula (15) takes the simplest form

$$
E_{k}=0,5 m\left(x_{t}^{2}+y_{t}^{2}\right)_{C}+0,5 \theta_{z, t}^{2} J_{C}^{z}
$$

Equation (16) can be extended to any spatial translational motion with rotation relative to one axis, since the $z t$ component is the same for all particles of the body,

$$
E_{k}=0,5 \int_{m}\left(x_{t}^{2}+y_{t}^{2}+z_{t}^{2}\right) \delta m=0,5\left[\int_{m}\left(x_{t}^{2}+y_{t}^{2}\right) \delta m+\int_{m}\left(z_{t}^{2}\right)_{C} \delta m\right]=0,5 m v_{C}^{2}+0,5 \theta_{z, t}^{2} J_{C}^{z}
$$

The result can be written in invariant form for any axis

$$
E_{k}=0,5 m v_{C}^{2}+0,5 \theta_{i, t}^{2} J_{C}^{i}
$$

but it cannot be used for the spatial motion of solids with rotation relative to 2 or 3 axes due to the lack of a method for determining the moment of inertia relative to the instantaneous axis of rotation $J_{C}^{i}$ when its position changes.

The exact analytical dependence of the kinetic energy in the spatial motion of a rigid body with arbitrary rotation can be obtained by representing the integral (10) as the sum of three integrals, in each of which the integrals represent the sum of the eponymous velocity components of the joint plane-parallel motions

$$
\begin{aligned}
& E_{k}=0,5 \int_{m} v^{2} \delta m=0,5 \int_{m}\left(x_{t}^{2}+y_{t}^{2}+z_{t}^{2}\right) \delta m= \\
& =0,5 \int_{m}\left(x_{t}^{\prime}+x_{t}^{\prime \prime}+x_{t}^{\prime \prime \prime}\right)^{2} \delta m+0,5 \int_{m}\left(y_{t}^{\prime}+y_{t}^{\prime \prime}+y_{t}^{\prime \prime \prime}\right)^{2} \delta m+0,5 \int_{m}\left(z_{t}^{\prime}+z_{t}^{\prime \prime}+z_{t}^{\prime \prime \prime}\right)^{2} \delta m,
\end{aligned}
$$

where the strokes indicate the velocity components from the three joint plane-parallel movements with a common pole: 1 stroke - rotation relative to the x-axis, 2 strokes-for the $y$-axis, 3 strokes-for the z-axis. This corresponds to the rule of geometric addition of velocities when joint movements, it is performed using the superposition principle (7).

To reduce the mathematical entries, we first consider the rotation of the body relative to the three coordinate axes passing through the fixed pole $P\left(\alpha_{P}, \beta_{P}\right)$. To the result obtained we add the energy of the translational motion of the body together with the pole, as provided for in Koenig's theorem.

From the above equations (3) - (6), it follows that in each integral of equation (18), one of the terms turns to 0 


$$
E_{k}=0,5 \int_{m}\left(x_{t}^{\prime \prime}+x_{t}^{\prime \prime \prime}\right)^{2} \delta m+0,5 \int_{m}\left(y_{t}^{\prime}+y_{t}^{\prime \prime \prime}\right)^{2} \delta m+0,5 \int_{m}\left(z_{t}^{\prime}+z_{t}^{\prime \prime}\right)^{2} \delta m
$$

For the first integral with the velocity components along the $x$-axis, taking into account equations (3) - (6), we obtain (the superscript with the designation $E_{k}^{i}$ shows the direction of the velocity component in this integral)

$$
E_{k}^{x}=0,5 \int_{m}\left\{\theta_{z, t}^{2}\left(y-\beta_{P}\right)^{2}+\theta_{y, t}^{2}\left(z-\gamma_{P}\right)^{2}-2 \theta_{y, t} \theta_{z, t}\left(y-\beta_{P}\right)\left(z-\gamma_{P}\right)\right\} \delta m .
$$

Since the angular velocities $\theta_{i, t}$ are the same for all the particles of the body, they can be extracted from the integral

$$
E_{k}^{x}=0,5 \theta_{z, t}^{2} \int_{m}\left(y-\beta_{P}\right)^{2} \delta m+0,5 \theta_{y, t}^{2} \int_{m}\left(z-\gamma_{P}\right)^{2} \delta m-\theta_{y, t} \theta_{z, t} \int_{m}\left(y-\beta_{P}\right)\left(z-\gamma_{P}\right) \delta m .
$$

The integrals remaining in the right part refer to the axial and centrifugal moments of inertia $[1,7]$

$$
\left(I_{y}^{z}\right)_{P}=\int_{m}\left(y-\beta_{P}\right)^{2} \delta m, \quad\left(I_{z}^{y}\right)_{P}=\int_{m}\left(z-\gamma_{P}\right)^{2} \delta m, \quad\left(I_{y z}\right)_{P}=\int_{m}\left(y-\beta_{P}\right)\left(z-\gamma_{P}\right) \delta m .
$$

For greater certainty, the upper index of the axial moments of inertia $\left(I_{i}^{j}\right)_{P}$ indicates the direction of the normal to the corresponding plane, which coincides with the axis of rotation indicated by the index of angular velocity $\theta_{i, t}$ in the term under consideration. For centrifugal moments of inertia, where the multipliers are two angular velocities, there are no upper indices. Lowercase subscripts at moments of inertia $\left(I_{i j}^{k}\right)_{P}$ in Equations and further indicate the variables in the corresponding integrand functions. Equation (21) takes the form

$$
E_{k}^{x}=0,5 \theta_{z, t}^{2}\left(I_{y}^{z}\right)_{P}+0,5 \theta_{y, t}^{2}\left(I_{z}^{y}\right)_{P}-\theta_{y, t} \theta_{z, t}\left(I_{y z}\right)_{P} .
$$

For the other two integrals of equation (19), converting them using equations (5) and (6) to the form (20), we find

$$
\begin{gathered}
E_{k}^{y}=0,5 \theta_{z, t}^{2}\left(I_{x}^{z}\right)_{P}+0,5 \theta_{x, t}^{2}\left(I_{z}^{x}\right)_{P}-\theta_{x, t} \theta_{z, t}\left(I_{x z}\right)_{P}, \\
E_{k}^{z}=0,5 \theta_{x, t}^{2}\left(I_{y}^{x}\right)_{P}+0,5 \theta_{y, t}^{2}\left(I_{x}^{y}\right)-\theta_{y, t} \theta_{x, t}\left(I_{x y}\right)_{P},
\end{gathered}
$$

Summing up the right-hand sides of equations (23) - (25), we get

$$
E_{k}=0,5 \theta_{z, t}^{2}\left(I_{x}^{z}+I_{y}^{z}\right)_{P}+0,5 \theta_{y, t}^{2}\left(I_{x}^{y}+I_{z}^{y}\right)_{P}+0,5 \theta_{x, t}^{2}\left(I_{y}^{x}+I_{z}^{x}\right)_{P}-\theta_{y, t} \theta_{z, t}\left(I_{y z}\right)_{P}-\theta_{x, t} \theta_{z, t}\left(I_{x z}\right)_{P}-\theta_{y, t} \theta_{x, t}\left(I_{x y}\right)_{P},
$$

where

$$
\begin{array}{rr}
\left(I_{x}^{y}\right)_{P}=\left(I_{x}^{z}\right)_{P}=\int_{m}\left(x-\alpha_{P}\right)^{2} \delta m, \quad\left(I_{y}^{x}\right)_{P}=\left(I_{y}^{z}\right)_{P}=\int_{m}\left(y-\beta_{P}\right)^{2} \delta m, \quad\left(I_{z}^{x}\right)_{P}=\left(I_{z}^{y}\right)_{P}=\int_{m}\left(z-\gamma_{P}\right)^{2} \delta m, \\
\left(I_{x y}\right)_{P}=\int_{m}\left(x-\alpha_{P}\right)\left(y-\beta_{P}\right) \delta m, \quad\left(I_{y z}\right)_{P}=\int_{m}\left(y-\beta_{P}\right)\left(z-\gamma_{P}\right) \delta m, & \left(I_{z x}\right)_{P}=\int_{m}\left(x-\alpha_{P}\right)\left(z-\gamma_{P}\right) \delta m .
\end{array}
$$

The sum of the axial moments of inertia in the first three terms of equation (26) coincides with those generally accepted in solid mechanics

$$
\begin{gathered}
\left(I_{y}^{z}\right)_{P}+\left(I_{x}^{z}\right)_{P}=\int_{m}\left[\left(x-\alpha_{P}\right)^{2}+\left(y-\beta_{P}\right)^{2}\right] \delta m=J_{P}^{z}, \\
\left(I_{z}^{y}\right)_{P}+\left(I_{x}^{y}\right)_{P}=\int_{m}\left[\left(x-\alpha_{P}\right)^{2}+\left(z-\gamma_{P}\right)^{2}\right] \delta m=J_{P}^{y},
\end{gathered}
$$




$$
\left(I_{y}^{x}\right)_{P}+\left(I_{z}^{x}\right)_{P}=\int_{m}\left[\left(z-\gamma_{P}\right)^{2}+\left(y-\beta_{P}\right)^{2}\right] \delta m=J_{P}^{x}
$$

These moments of inertia can be determined by both by the current and the initial configuration of the body, since the integrand functions, as follows from the equations of motion (3) - (6), retain their values at any rotation of the body, for example

$$
J_{P}^{z}=\int_{m}\left[\left(x-\alpha_{P}\right)^{2}+\left(y-\beta_{P}\right)^{2}\right] \delta m=\int_{m}\left[\left(\alpha-\alpha_{P}\right)^{2}+\left(\beta-\beta_{P}\right)^{2}\right] \delta m=\text { const }
$$

Taking into account the generally accepted notation (30) instead of (28), we get

$$
E_{k}=0,5 \theta_{z, t}^{2} J_{P}^{z}+0,5 \theta_{y, t}^{2} J_{P}^{y}+0,5 \theta_{x, t}^{2} J_{P}^{x}-\theta_{y, t} \theta_{z, t}\left(I_{y z}\right)_{P}-\theta_{x, t} \theta_{z, t}\left(I_{x z}\right)_{P}-\theta_{y, t} \theta_{x, t}\left(I_{x y}\right)_{P} .
$$

By analogy with equation (1), the upper index of the moments of inertia $J_{P}^{i}$ corresponds to the axis relative to which it should be determined. The subscript indicates the point through which the axis of rotation should pass $i \in(x, y, z)$.

Using the equations of motion (3) - (6), the system (27) for the centrifugal moments of inertia $\left(I_{i j}\right) P$ can be converted to the form

$$
\begin{gathered}
\left(I_{x y}\right)_{P}=\int_{m}\left(x-\alpha_{P}\right)\left(y-\beta_{P}\right) \delta m=0,5 \sin \left(2 \Delta \theta_{z}\right) \int_{m}\left[\left(\alpha-\alpha_{P}\right)^{2}-\left(\beta-\beta_{P}\right)^{2}\right] \delta m+\cos \left(2 \Delta \theta_{z}\right) \int_{m}\left(\alpha-\alpha_{P}\right)\left(\beta-\beta_{P}\right) \delta m, \\
\left(I_{y z}\right)_{P}=\int_{m}\left(y-\beta_{P}\right)\left(z-\gamma_{P}\right) \delta m=0,5 \sin \left(2 \Delta \theta_{x}\right) \int_{m}\left[\left(\beta-\beta_{P}\right)^{2}-\left(\gamma-\gamma_{P}\right)^{2}\right] \delta m+\cos \left(2 \Delta \theta_{x}\right) \int_{m}\left(\beta-\beta_{P}\right)\left(\gamma-\gamma_{P}\right) \delta m \\
\left(I_{z x}\right)_{P}=\int_{m}\left(x-\alpha_{P}\right)\left(z-\gamma_{P}\right) \delta m=0,5 \sin \left(2 \Delta \theta_{y}\right) \int_{m}\left[\left(\gamma-\gamma_{P}\right)^{2}-\left(\alpha-\alpha_{P}\right)^{2}\right] \delta m+\cos \left(2 \Delta \theta_{y}\right) \int_{m}\left(\alpha-\alpha_{P}\right)\left(\gamma-\gamma_{P}\right) \delta m
\end{gathered}
$$

The resulting equations are similar to the known relations for the change in the moments of inertia of solids when the coordinate axes are rotated [1-2]. Consequently, the last three terms in equation (30) take into account the change in the centrifugal moments of inertia (27) relative to the axes of the observer's coordinate system due to the rotation of the body.

Using the notation for the moments of inertia in the initial state

$$
\begin{array}{r}
\left(I_{\alpha}\right)_{P}=\int_{m}\left(\alpha-\alpha_{P}\right)^{2} \delta m, \quad\left(I_{\beta}\right)_{P}=\int_{m}\left(\beta-\beta_{P}\right)^{2} \delta m, \quad\left(I_{\gamma}\right)_{P}=\int_{m}\left(\gamma-\gamma_{P}\right)^{2} \delta m \\
\left(I_{\alpha \beta}\right)_{P}=\int_{m}\left(\alpha-\alpha_{P}\right)\left(\beta-\beta_{P}\right) \delta m, \quad\left(I_{\beta \gamma}\right)_{P}=\int_{m}\left(\beta-\beta_{P}\right)\left(\gamma-\gamma_{P}\right) \delta m,\left(I_{\alpha \gamma}\right)_{P}=\int_{m}\left(\alpha-\alpha_{P}\right)\left(\gamma-\gamma_{P}\right) \delta m
\end{array}
$$

instead of equations (31), we get

$$
\begin{gathered}
\left(I_{x y}\right)_{P}=0,5 \sin \left(2 \Delta \theta_{z}\right)\left[\left(I_{\alpha}\right)_{P}-\left(I_{\beta}\right)_{P}\right]+\cos \left(2 \Delta \theta_{z}\right)\left(I_{\alpha \beta}\right)_{P}, \\
\left(I_{y z}\right)_{P}=0,5 \sin \left(2 \Delta \theta_{x}\right)\left[\left(I_{\beta}\right)_{P}-\left(I_{\gamma}\right)_{P}\right]+\cos \left(2 \Delta \theta_{x}\right)\left(I_{\beta \gamma}\right)_{P}, \\
\left(I_{z x}\right)_{P}=0,5 \sin \left(2 \Delta \theta_{y}\right)\left[\left(I_{\gamma}\right)_{P}-\left(I_{\alpha}\right)_{P}\right]+\cos \left(2 \Delta \theta_{y}\right)\left(I_{\alpha \gamma}\right)_{P} .
\end{gathered}
$$

To distinguish the axial and centrifugal moments of inertia $\left(I_{i j}\right)_{P}$ in the current and initial states, the Lagrange variables $\alpha_{P} \in(\alpha, \beta, \gamma)$ are used for the lower indices of the initial state in the right part of equations (32) and (33). 
Thus, the kinetic energy of a solid body during its arbitrary rotation relative to a fixed pole $P\left(\alpha_{P}, \beta_{P}\right)$ can be found in the most general case by equation (30). The moments of inertia (28) remain constant at all rotations of the body in accordance with equation (29). The centrifugal moments (27) change when the body rotates and can be calculated through the moments of inertia (32) in accordance with equations (33).

If we combine the pole $P$ with the center of mass $C$, equation (30) takes the form

$$
E_{k}=0,5\left(\theta_{z, t}^{2} J_{C}^{z}+\theta_{y, t}^{2} J_{C}^{y}+\theta_{x, t}^{2} J_{C}^{x}\right)-\theta_{y, t} \theta_{z, t}\left(I_{y z}\right)_{C}-\theta_{x, t} \theta_{z, t}\left(I_{x z}\right)_{C}-\theta_{y, t} \theta_{x, t}\left(I_{x y}\right)_{C},
$$

where for the axial moments of inertia instead of (28) we get

$$
J_{C}^{z}=\int_{m}\left[\left(x-\alpha_{C}\right)^{2}+\left(y-\beta_{C}\right)^{2}\right] \delta m, \quad J_{C}^{y}=\int_{m}\left[\left(x-\alpha_{C}\right)^{2}+\left(z-\gamma_{C}\right)^{2}\right] \delta m, \quad J_{C}^{x}=\int_{m}\left[\left(y-\beta_{C}\right)^{2}+\left(z-\gamma_{C}\right)^{2}\right] \delta m .
$$

For centrifugal moments, instead of (33, the equations are valid

$$
\begin{gathered}
\left(I_{x y}\right)_{C}=0,5 \sin \left(2 \Delta \theta_{z}\right)\left[\left(I_{\alpha}\right)_{C}-\left(I_{\beta}\right)_{C}\right]+\cos \left(2 \Delta \theta_{z}\right)\left(I_{\alpha \beta}\right)_{C}, \\
\left(I_{y z}\right)_{C}=0,5 \sin \left(2 \Delta \theta_{x}\right)\left[\left(I_{\beta}\right)_{C}-\left(I_{\gamma}\right)_{C}\right]+\cos \left(2 \Delta \theta_{x}\right)\left(I_{\beta \gamma}\right)_{C}, \\
\left(I_{z x}\right)_{C}=0,5 \sin \left(2 \Delta \theta_{y}\right)\left[\left(I_{\gamma}\right)_{C}-\left(I_{\alpha}\right)_{C}\right]+\cos \left(2 \Delta \theta_{y}\right)\left(I_{\alpha \gamma}\right)_{C} .
\end{gathered}
$$

Equations (30) for an arbitrary pole and (34) for a pole at the center of mass, in contrast to (15) and (16), coincide in form, since the assumption is used $\left(x_{t}\right)_{P}=\left(y_{t}\right)_{P}=0$.

To calculate the kinetic energy, taking into account the translational motion of the body with the velocity of the center of mass, equations (30) and (34), in accordance with Koenig's theorem, are converted to the form

$$
\begin{aligned}
& E_{k}=0,5 v_{P}^{2} m+0,5\left(\theta_{z, t}^{2} J_{P}^{z}+\theta_{y, t}^{2} J_{P}^{y}+\theta_{x, t}^{2} J_{P}^{x}\right)-\theta_{y, t} \theta_{z, t}\left(I_{y z}\right)_{P}-\theta_{x, t} \theta_{z, t}\left(I_{x z}\right)_{P}-\theta_{y, t} \theta_{x, t}\left(I_{x y}\right)_{P}, \\
& E_{k}=0,5 v_{C}^{2} m+0,5\left(\theta_{z, t}^{2} J_{C}^{z}+\theta_{y, t}^{2} J_{C}^{y}+\theta_{x, t}^{2} J_{C}^{x}\right)-\theta_{y, t} \theta_{z, t}\left(I_{y z}\right)_{C}-\theta_{x, t} \theta_{z, t}\left(I_{x z}\right)_{C}-\theta_{y, t} \theta_{x, t}\left(I_{x y}\right)_{C} .
\end{aligned}
$$

The axial moments of inertia determine the equations (28) or (35), the centrifugal (33) or (36).

The choice of the pole in equation (37) is unambiguously determined at the stage of kinematic analysis, since equations (3) - (6) assume its motion to be known, and it must belong to the kinematic pairs connecting the slave and the master links. However, when calculating the generalized forces, it is more convenient to use equation (38) with the kinematic characteristics of the center of mass [8].

\section{Discussion}

The accuracy of equations (30) and (34) can be estimated by comparison with the results of numerical integration, using equation (10) and the system of equations of joint motion of type (9) obtained using the superposition principle [4-5].

For example, when joint rotations relative to the $z$ and $x$ axes, if we consider the rotation relative to the $z$ axis to be external, taking into account equations (3) - (5), we obtain

$$
\begin{gathered}
x=\alpha_{P}+\left(\alpha-\alpha_{P}\right) \cos \Delta \theta_{z}-\left[\left(\beta-\beta_{P}\right) \cos \Delta \theta_{x}-\left(\gamma-\gamma_{P}\right) \sin \Delta \theta_{x}\right] \sin \Delta \theta_{z}, \\
y=\beta_{P}+\left(\alpha-\alpha_{P}\right) \sin \Delta \theta_{z}+\left[\left(\beta-\beta_{P}\right) \cos \Delta \theta_{x}-\left(\gamma-\gamma_{P}\right) \sin \Delta \theta_{x}\right] \cos \Delta \theta_{z}, \\
z=\gamma_{P}+\left(\beta-\beta_{P}\right) \sin \Delta \theta_{x}+\left(\gamma-\gamma_{P}\right) \cos \Delta \theta_{x} .
\end{gathered}
$$

The velocity components of the joint motion (39) can be written in terms of Lagrange or Euler variables, and in the latter case they have a more compact form, for example 


$$
y_{t}=\theta_{z, t}\left(x-\alpha_{P}\right)-\theta_{x, t}\left(z-\gamma_{P}\right) \cos \Delta \theta_{z} .
$$

However, for subsequent integration, it is preferable to keep the Lagrange variables as arguments

$$
\begin{gathered}
x_{t}=\theta_{z, t}\left\{-\left(\alpha-\alpha_{P}\right) \sin \Delta \theta_{z}-\left[\left(\beta-\beta_{P}\right) \cos \Delta \theta_{x}-\left(\gamma-\gamma_{P}\right) \sin \Delta \theta_{x}\right] \cos \Delta \theta_{z}\right\}+ \\
+\theta_{x, t}\left\{\left[\left(\beta-\beta_{P}\right) \sin \Delta \theta_{x}+\left(\gamma-\gamma_{P}\right) \cos \Delta \theta_{x}\right] \sin \Delta \theta_{z}\right\},
\end{gathered}
$$

$$
y_{t}=\theta_{z, t}\left\{\left(\alpha-\alpha_{P}\right) \cos \Delta \theta_{z}-\left[\left(\beta-\beta_{P}\right) \cos \Delta \theta_{x}-\left(\gamma-\gamma_{P}\right) \sin \Delta \theta_{x}\right] \sin \Delta \theta_{z}\right\}-\theta_{x, t}\left[\left(\beta-\beta_{P}\right) \sin \Delta \theta_{x}+\left(\gamma-\gamma_{P}\right) \cos \Delta \theta_{x}\right] \cos \Delta \theta_{z},
$$

$$
z_{t}=\theta_{x, t}\left[\left(\beta-\beta_{P}\right) \cos \Delta \theta_{x}-\left(\gamma-\gamma_{P}\right) \sin \Delta \theta_{x}\right]
$$

You can make sure that the equations for coordinates (39) and velocities (40) are converted to the corresponding initial equations (3) - (6), if you exclude rotation relative to one of the axes in the joint motion.

The integrand function of equation (10), taking into account (40), takes the form

$$
\begin{gathered}
x_{t}{ }^{2}+y_{t}{ }^{2}+z_{t}{ }^{2}=\theta_{z, t}{ }^{2}\left(\alpha-\alpha_{P}\right)^{2}+\theta_{z, t}{ }^{2}\left[\left(\beta-\beta_{P}\right) \cos \Delta \theta_{x}-\left(\gamma-\gamma_{P}\right) \sin \Delta \theta_{x}\right]^{2}+ \\
+\theta_{x, t}{ }^{2}\left[\left(\beta-\beta_{P}\right)^{2}+\left(\gamma-\gamma_{P}\right)^{2}\right]-2 \theta_{x, t} \theta_{z, t}\left(\alpha-\alpha_{P}\right) *\left[\left(\beta-\beta_{P}\right) \sin \Delta \theta_{x}+\left(\gamma-\gamma_{P}\right) \cos \Delta \theta_{x}\right] .
\end{gathered}
$$

Numerical verification shows the coincidence of the values of the integrand functions (41) and their sum in equation (19) at any point of the body at any values of angular velocities, therefore, the integration results should be the same.

In particular cases of rotation relative to one of the two axes under consideration, the integration results coincide with the generally accepted ones:

when rotating relative to the $x$-axis

$$
E_{k}=0,5 \int_{m}\left(x_{t}^{2}+y_{t}^{2}+z_{t}^{2}\right) \delta m=0,5 \theta_{x, t}^{2} \int_{m}\left[\left(\beta-\beta_{P}\right)^{2}+\left(\gamma-\gamma_{P}\right)^{2}\right] \delta m=0,5 \theta_{x, t}^{2} \int_{m}\left[\left(y-y_{P}\right)^{2}+\left(z-z_{P}\right)^{2}\right] \delta m=0,5 \theta_{x, t}^{2} J_{P}^{x},
$$

when rotating relative to the $z$-axis

$$
E_{k}=0,5 \int_{m}\left(x_{t}^{2}+y_{t}^{2}+z_{t}^{2}\right) \delta m=0,5 \theta_{z, t}^{2} \int_{m}\left[\left(\alpha-\alpha_{P}\right)^{2}+\left(\beta-\beta_{P}\right)^{2}\right] \delta m=0,5 \theta_{z, t}^{2} \int_{m}\left[\left(x-x_{P}\right)^{2}+\left(y-y_{P}\right)^{2}\right] \delta m=0,5 \theta_{z, t}^{2} J_{P}^{z} .
$$

However, for the joint motion (39), it is not possible to present the integration result in an analytical form.

Separately, we note that in the superposition of several rotational movements, the final form of the equations depends on the sequence of operations performed and the choice of external or internal movements. This method is only useful when comparing the results of numerical and analytical methods for determining the kinetic energy.

\section{Conclusions}

Thus, equations (37) and (38) determine the kinetic energy of a solid body in the most general case of spatial motion with arbitrary rotation and can be used for dynamic analysis of mechanisms of any complexity with known information about the current values of linear and angular velocities. The centrifugal moments of inertia included in these equations must be determined from the initial position of the body, their change due to rotation takes into account the last three terms.

For rotation relative to a single axis, such as the $z$ axis, the results of equations (38) and (1) are the same

$$
E_{k}=0,5 v_{C}^{2} m+0,5 \theta_{z, t}^{2}\left(I_{y}+I_{x}\right)_{C}=0,5 v_{C}^{2} m+0,5 \theta_{z, t}^{2} J_{C}^{z} .
$$

For the rotation considered above with respect to the two axes $x$ and $z$, the result differs from Koenig's formula (1) by the last term with the centrifugal moment, 


$$
E_{k}=0,5 v_{P}^{2} m+0,5 \theta_{z, t}^{2} J_{P}^{z}+0,5 \theta_{x, t}^{2} J_{P}^{x}-\theta_{x, t} \theta_{z, t} I_{x z}
$$

The structure of the obtained equations does not allow us to propose an alternative method for the analytical determination of the moment of inertia relative to the instantaneous axis of rotation when its position changes, which can only be determined experimentally [1].

Given the sign of the new terms, it can be assumed that the calculated results according to the Koenig formula (1) exceed their actual values. The error depends on the complexity of the control program and the design features of the mechanism under study.

\section{References}

1. Zhuravlev V. F. Fundamentals of theoretical mechanics. - M.: MIPT, 2008. - 304 p.

2. Gernet M. M. Course of theoretical mechanics. - M.: Higher school, 1987. - 344 p.

3. Alyushin Yu. A. Energeticheskie osnovy mekhaniki. - M.: Mashinostroenie, 1999. - 192 p.

4. Alyushin Yu. A. Solid state mechanics in Lagrange variables. - M.: Mashinostroenie, 2012. -192 p.

5. Alyushin Yu. A. The principle of superposition of motions in the space of Lagrange variables//Problems of mechanical engineering and reliability of machines. - 2001. - No. 3. p. 13-19.

6. Alyushin Yu. A. Energeticheskie osnovy mekhaniki. - Lambert Academic Publishing, 2016. - 281 c.

7. Rabotnov Yu. N. Mechanics of a deformable solid body. - M.: Nauka, 1979. -744 p.

8. Alyushin Yu. A., Verzhansky P. M. Structural, kinematic and dynamic analysis of lever mechanisms. - M.: MISIS, 2015. - 104 p. 